\title{
ADAM22/LGI1 complex as a new actionable target for breast cancer brain metastasis
}

\author{
Sara Charmsaz ${ }^{1 \dagger}$, Ben Doherty ${ }^{1 \dagger}$, Sinéad Cocchiglia', Damir Varešlija ${ }^{1}$, Attilio Marino², Nicola Cosgrove', \\ Ricardo Marques ${ }^{1}$, Nolan Priedigkeit ${ }^{3}$, Siobhan Purcell', Fiona Bane ${ }^{1}$, Jarlath Bolger ${ }^{1}$, Christopher Byrne', \\ Philip J. O'Halloran ${ }^{4}$, Francesca Brett ${ }^{5}$, Katherine Sheehan ${ }^{6}$, Kieran Brennan ${ }^{7}$ Ann M. Hopkins ${ }^{7}$, Stephen Keelan ${ }^{1}$, \\ Petra Jagust ${ }^{1}$, Stephen Madden ${ }^{8}$, Chiara Martinelli ${ }^{2}$, Matteo Battaglini ${ }^{2,9}$, Steffi Oesterreich ${ }^{3}$, Adrian V. Lee ${ }^{3}$, \\ Gianni Ciofani ${ }^{2}$, Arnold D. K. Hill ${ }^{7,10}$ and Leonie S. Young ${ }^{1 *}$
}

\begin{abstract}
Background: Metastatic breast cancer is a major cause of cancer-related deaths in woman. Brain metastasis is a common and devastating site of relapse for several breast cancer molecular subtypes, including oestrogen receptor-positive disease, with life expectancy of less than a year. While efforts have been devoted to developing therapeutics for extra-cranial metastasis, drug penetration of blood-brain barrier (BBB) remains a major clinical challenge. Defining molecular alterations in breast cancer brain metastasis enables the identification of novel actionable targets.

Methods: Global transcriptomic analysis of matched primary and metastatic patient tumours ( $n=35$ patients, 70 tumour samples) identified a putative new actionable target for advanced breast cancer which was further validated in vivo and in breast cancer patient tumour tissue ( $n=843$ patients). A peptide mimetic of the target's natural ligand was designed in silico and its efficacy assessed in in vitro, ex vivo and in vivo models of breast cancer metastasis.

Results: Bioinformatic analysis of over-represented pathways in metastatic breast cancer identified ADAM22 as a top ranked member of the ECM-related druggable genome specific to brain metastases. ADAM22 was validated as an actionable target in in vitro, ex vivo and in patient tumour tissue ( $n=843$ patients). A peptide mimetic of the ADAM22 ligand LGI1, LGI1MIM, was designed in silico. The efficacy of LGI1MIM and its ability to penetrate the BBB were assessed in vitro, ex vivo and in brain metastasis BBB 3D biometric biohybrid models, respectively. Treatment with LGI1MIM in vivo inhibited disease progression, in particular the development of brain metastasis.
\end{abstract}

Conclusion: ADAM22 expression in advanced breast cancer supports development of breast cancer brain metastasis. Targeting ADAM22 with a peptide mimetic LGI1MIM represents a new therapeutic option to treat metastatic brain disease.

Keywords: Breast cancer metastases, Brain metastases, ADAM22, LGI1, ECM signalling, Blood-brain barrier, Targeted therapy

\footnotetext{
* Correspondence: lyoung@rcsi.ie

'Sara Charmsaz and Ben Doherty contributed equally to this work.

${ }^{1}$ Endocrine Oncology Research Group, Department of Surgery, Royal College

of Surgeons in Ireland, Dublin 2, Ireland

Full list of author information is available at the end of the article
}

C The Author(s). 2020 Open Access This article is licensed under a Creative Commons Attribution 4.0 International License, which permits use, sharing, adaptation, distribution and reproduction in any medium or format, as long as you give appropriate credit to the original author(s) and the source, provide a link to the Creative Commons licence, and indicate if changes were made. The images or other third party material in this article are included in the article's Creative Commons licence, unless indicated otherwise in a credit line to the material. If material is not included in the article's Creative Commons licence and your intended use is not permitted by statutory regulation or exceeds the permitted use, you will need to obtain permission directly from the copyright holder. To view a copy of this licence, visit http://creativecommons.org/licenses/by/4.0/. The Creative Commons Public Domain Dedication waiver (http://creativecommons.org/publicdomain/zero/1.0/) applies to the data made available in this article, unless otherwise stated in a credit line to the data. 


\section{Background}

Breast cancer metastasis is responsible for the vast majority of all breast cancer related deaths [1]. Although overall clinical molecular subtype switching on metastasis is rare, global alterations in the transcriptome are known to facilitate disease progression. Longitudinal sequencing studies of matched primary and metastatic tumours define transcriptional gains in tyrosine kinase and extracellular matrix (ECM)/cell-to-cell communication signalling networks [2, 3], which may represent acquired vulnerabilities and open up new therapeutic opportunities. A disintegrin and metalloproteinases (ADAMs) and the related ADAM with thrombospondin motifs (ADAM TS) are key members of the ECM signalling network [4, 5]. ADAMs are a family of multi-domain transmembrane proteins $[6,7]$, with functions in cell adhesion, migration and proteolysis [8]. Approximately half the family members have matrix metalloproteinase-like activity, with several members, including ADAM10 and 17 being targeted for cancer therapy [9].

We have previously demonstrated a role for ADAM22 in endocrine-resistant breast cancer and identified ADAM22 as an independent predictor of poor diseasefree survival [10]. Furthermore, recent studies by Li et al. [11] reported upregulation of ADAM22 through decreased miR-449a, resulting in the development of tamoxifen resistance in ER-positive breast cancer cells. These studies suggest a role for ADAM22 in the development of endocrine-resistant disease and metastatic progression [10]. Unlike other family members implicated in cancer, ADAM22 lacks a functional metalloproteinase domain [12] and may mediate its pro-tumourigenic effects through interaction with other cell surface tyrosine kinase receptors using its EGF-like domain [6].

At a physiological level, ADAM22 acts as a receptor on the surface of the postsynaptic neuron to regulate signal transmission through binding to leucine-rich, glioma inactivated gene 1 (LGI1), a neuronal protein and a specific ligand for ADAM22 [12]. The LGI1/ADAM22 complex has been reported to have a role in epilepsy, and the ligand/receptor has been suggested as a therapeutic target for synaptic disorders [13]. In cancer, LGI1 has been shown to function as a tumour suppressor gene for glioblastoma and neuroblastoma [14, 15], and we have previously reported LGI1-targeted inhibition of cell migration in ER-positive endocrine-resistant breast cancer cells mediating distant metastatic competency [10].

Breast cancer is the second most common primary tumour type that spreads to the brain and affects approximately $10 \%$ of ER-positive breast cancer patients [16]. Metastasis to the brain confers particularly poor prognosis with life expectancy following diagnosis of less than a year. Currently, the standard therapeutic for the vast majority of breast cancer brain metastasis patients includes surgery, radiotherapy and in some cases systemic chemotherapy, all which have limited success [16]. Identifying new therapeutics for breast cancer brain metastasis, which can target brain colonising cells and can cross the blood-brain barrier, is required. Here we investigate the role of ADAM22 in ER-positive breast cancer brain metastasis and evaluate the potential of targeting ADAM22 with the small peptide mimetic of LGI1 as a new therapeutic strategy to treat brain metastasis.

\section{Methods}

Ethics

All clinical material was collected under the clinical trial NCT01840293 (https://clinicaltrials.gov) following ethical approval from Beaumont Hospital Medical Research Ethics Committee.

\section{Cell culture}

The MCF7 cells were obtained from ATCC and cultured in Minimum Essential Medium Eagle (MEM) (M2279, Sigma) supplemented with $2 \mathrm{mM}$ L-glutamine (G7513, Sigma) and 10\% fetal calf serum (FCS) (F7524, Sigma). The LY2 cells were a gift from Robert Clarke (Georgetown, USA) and were cultured as previously described [17]. Each cell line was tested for mycoplasma contamination (LT07-118, Lonza) and genotyped (Source BioScience) according to ATCC guidelines. T347 cells were derived from an ER+ PR- HER2+ brain metastatic tumour from a breast cancer patient as previously described [18]. AI resistant LetR cells were generated as previously described [19].

\section{Bioinformatics}

\section{Differential gene expression-exome capture RNA} sequencing of brain and bone metastasis

Sequencing reads from previously published exome capture sequencing of patient-matched primary breast tumour with bone metastases [20] and brain metastases [3] were mapped against human reference transcriptome GRCh38.p10 using Salmon v.0.9.1 [21]. Gene level summarised counts were used as input for differential gene expression. DESeq2 [22] was used to identify differential gene expression separately in primary breast tumours compared to matched brain metastases and matched bone metastases. A multi-factor design was used for matched (gene $e_{i} \sim$ patient + tumour). A log2 fold change of greater than \pm 1.5 and an adjusted $p$ value of $<0.05$ were used to identify up- or downregulated genes. EnhancedVolcano was used to generate volcano plots of differential gene expression [23], with genes upregulated in metastasis annotated by Matrisome database version 2.0 [23] labelled. 


\section{Differential gene expression-RNA sequencing of liver metastasis}

Raw sequencing reads [2] were processed using fastQC, bbmap and subreads to obtain normalised gene counts for 3 patient-matched primary breast with liver metastases including 2 matched nodal samples. Differential gene expression testing was performed using edgeR [24] (log2 fold change \pm 1.5 FDR $<0.05$ ).

\section{Gene Ontology Enrichment Testing}

The clusterProfiler package in $\mathrm{R}$ version 3.5.2 was used to functionally annotate genes according to Gene Ontology biological process (BP) categories using gene symbol ids as input [25]. Statistically significant differential genes identified from breast cancer brain, bone and liver metastases were treated as individual gene clusters for CompareCluster function. The hypergeometric test was used with significant GO terms for each cluster called based on a $q$-value $<0.05$. Significant GO terms were visualised using the dotplot function.

\section{Gene expression gains in druggable genome}

DESeq2 [22] was used to identify differential gene expression in ER-positive primary breast tumours compared to matched brain metastases using salmon gene counts from a previously published dataset [3]. A multifactor design was used for matched samples (gene $\mathrm{i}_{\mathrm{i}} \sim \mathrm{pa}-$ tient + tumour). A $\log 2$ fold change of greater than \pm 1.5 and an adjusted $p$ value of $<0.05$ were used to identify up- or downregulated genes. In order to identify extracellular matrix (ECM)-related genes upregulated in ERpositive primary breast tumours versus brain metastases, genes were cross referenced against the Matrisome database version 2.0 [23]. Druggable genome categorised genes $(n=6106)$ were downloaded from the Drug-Gene Interaction database version 3.0 (DGIdb 3.0) [26]. ECMrelated genes were further cross-referenced against "druggable genome" genes. Identification of recurrent gene expression gains were performed as per Vareslija et al. [3]. After assignment of discrete expression gains in ECM druggable genome genes, those genes with gains in greater than 3 patients were plotted using the oncoprint function in ComplexHeatmap [27].

\section{Association of ADAM22 with brain predominant marker}

A gene-gene Pearson correlation coefficient for known brain predominant genes $[28,29]$ and ADAM22 in breast cancer brain metastatic tumours $(N=21$ patients) was undertaken. Correlation score of $p<0.05$ was considered significantly correlated.

\section{CRISPR/Cas9 and lentiviral transduction}

Full details can be found in supplementary methods (Additional file 1). Briefly, ADAM22 was knocked out in LY2 cells using CRISPR/Cas9 technology (Additional file 2 Fig. S1 a-e) and ADAM22 was over-expressed with lentiviral particles (Additional file 2 Fig. S2 a-d).

\section{Mammosphere forming, anchorage independence and cell migration assays}

Functional assays were performed in the MCF7, LY2, LY2 ADAM22 KO, LY2 ADAM22 KI, LETR and T347 cells and were carried out in the presence of 4-OHT $\left[10^{-7} \mathrm{M}\right]$ where appropriate. Mammosphere culture and analysis was performed as previously described [30]. Anchorage independence was analysed using the agarose colony formation assay as previously described [31]. Cell migration was carried out using the Cellomics Cell Motility Kit (K0800011, Thermo Scientific) as previously described [32].

\section{Reverse phase proteomic study (RPPA)}

RPPA analysis was carried out on protein lysates from LY2 and LY2 ADAM22 KO using a Genetix QArray2 spotter (Additional file 2 Fig. S3a). Full details can be found in Supplementary methods.

\section{Immunohistochemistry (IHC)}

ADAM22 expression was examined on a tissue microarray (TMA) of breast cancer samples in a cohort of patients diagnosed from 2008 to 2015 from Beaumont Hospital, Dublin $(n=843)$ as previously described [33]. The TMA was immunostained using C-2 monoclonal ADAM22 antibody (sc-373,931, Santa Cruz) $(8 \mu \mathrm{g} / \mathrm{ml})$ and scored using the histoscore method. Matched primary and metastatic tissues were from patients from the above cohort, and healthy normal tissue sections from the brain, lung, liver, heart, spleen and kidney were obtained from Beaumont Hospital and scored using the histoscore method.

\section{Peptide synthesis}

Computer modelling was used to model ADAM22/ LGI1MIM interactions; details are provided in Supplementary methods. A 21 a.a. peptide mimetic of the ADAM22 disintegrin binding domain of LGI1 (LGI1MIM) was designed using I-TASSER software with a single cysteine to serine substitution (a.a. 447). Full length LGI1 and the peptide mimetic LGI1 was synthesised to $>95 \%$ purity by JPT Peptide Technology, Berlin.

\section{Organoid culture}

Organoid culture was generated from brain metastatic breast patient-derived sample (T347) following Sachs et al.'s protocol [34]. Briefly, frozen pieces $\left(3-6 \mathrm{~mm}^{3}\right)$ were minced, enzymatically digested with collagenase $(1 \mathrm{mg} / \mathrm{ml}$, Sigma, C9407) for $1.5 \mathrm{~h}$ at $37^{\circ} \mathrm{C}$ followed by mechanical digestion. Ten thousand single cells $(70 \mu \mathrm{m}$ strained) were 
seeded in organoid media [34] with $5 \%$ of Cultrex ${ }^{\circ}$ Reduced Growth Factor Basement Membrane Matrix, type 2 (BME, Trevigen, 3533-001-02). Once formed, organoids were treated with vehicle (0.1\% DMSO) or LGI1 $(25 \mathrm{nM})$. Each treatment was tested in multiple replicates $(>5)$. Cell viability was measured 7 days post treatment using CellTiter-Glo ${ }^{\circ}$ 3D Cell Viability assay (Promega) as per the manufacturer's protocol.

\section{Ex vivo assay and Ki67 staining}

Patient breast cancer brain metastatic ER-positive tumours (T347, T2447 and T328) and triple-negative tumour (T2203) were expanded in NOD/SCID mice [3]. The primary tumours were resected, grown on gelatin sponges (Spongostan, Johnson and Johnson) as previously described [33] and treated with oestrogen in the presence of LGI1 peptide mimetic (LGI1MIM) or vehicle for $72 \mathrm{~h}$. Following treatment, tumour pieces were formalin fixed and paraffin embedded for IHC analysis. The viability of the tumours was evaluated by screening for necrosis of the tissue and assessed for Ki67 by IHC to confirm viable, proliferating cells.

\section{Statistics}

Statistical analysis was performed using Prism (GraphPad, San Diego, CA, USA) and Stata software (StataCorp, College Station, TX, USA). Survival times between groups were calculated using log rank for quality of survival and compared with a $X^{2}$ distribution with 1 degree of freedom. Multivariate analysis for ADAM22 was carried out using Cox proportional hazards, modelled with PR status, HER2 status and node using the Breslow method for ties. Other appropriate statistical tests for each experiment were undertaken as described in the relevant "Results" section. Values of $p \leq 0.05$ were considered significant.

\section{In vivo experiments}

All animal experiments were performed in accordance with the European Communities Council Directive (2010/63/EU) and were reviewed and approved by Research Ethics Committee, RCSI under licence from the Department of Health and The Health Products Regulatory Authority (SI No543 2012). Luciferase-tagged, LY2, LY2 ADAM22 KO cells and LY2 ADAM22 KI cells (Additional file 2 Fig. S3 b-d) were injected into the mammary fat-pad of NOD/SCID mice. Full details are available in Supplementary methods.

\section{Biomimetic LGI1MIM-loaded liposome (LGI1MIM-LS) preparation, functionalisation and staining}

LGI1MIM-LSs were obtained using the thin-film evaporation method as described in Supplementary methods. LGI1MIM-LSs were functionalised with a streptavidine- conjugated anti-transferrin receptor antibody (anti-TfR; $25 \mu \mathrm{g} / \mathrm{ml}$ ) and purified [35]. For the evaluation of bloodbrain barrier (BBB) crossing, $5 \mathrm{mg} / \mathrm{ml}$ of LSs were stained with Vybrant ${ }^{\text {tw }}$ DiO labelling solution (1:25 dilution) and purified. LGI1MIM-LSs are characterised by unilamellar morphology and homogeneous size distribution of $100 \mathrm{~nm}$ diameter (Additional file 2 Fig. S4a).

\section{LGI1MIM-LS imaging and characterisation}

Transmission electron microscopy (TEM) analyses were carried out with a transmission electron microscope (JEOL 1011, Tokyo, JAPAN). Size and Z-potential distributions of a $100 \mu \mathrm{g} / \mathrm{ml}$ LGI1MIM-LSs dispersion were analysed using a Zetasizer Nano ZSP (Malvern Instrument). Full details are available in Supplementary methods.

\section{Multicellular blood-brain barrier (BBB) in vitro model}

BBB model was obtained by culturing the brain-derived endothelioma bEnd.3 cells (ATCC ${ }^{\circ}$ CRL-2299 $^{\text {tw }}$ at $8 \times$ $10^{4}$ cells $/ \mathrm{cm}^{2}$ ) and the C8D1A brain astrocytes (ATCC CRL-2541 $1^{\mathrm{mm}}$ at $2 \times 10^{4}$ cells $/ \mathrm{cm}^{2}$ ) on the luminal and abluminal side of $3 \mu \mathrm{m}$ porous transwells (Corning Incorporated). Expression of tight junction proteins and BBB crossing were assessed as described in Supplementary methods.

\section{Evaluation of LGI1MIM-LSs internalisation, Ki-67 expression and cell cycle analysis in T347 cells} Abluminal compartments were transferred to 24-well plates where T347 cells were seeded $24 \mathrm{~h}$ prior to the start of the experiment. $500 \mu \mathrm{g} / \mathrm{ml}$ LGI1MIM-LSs was added to the luminal compartment and incubated for $72 \mathrm{~h}$ (Additional file 2 Fig. S4). Liposome uptake, cell viability, Ki-67 expression and cell cycle analysis were investigated in the T347 cells cultured in the abluminal compartment. 3D CLSM of DiO-stained LGI1MIM-LSs internalised in T347 cells was performed using a C2s system (Nikon).

\section{WST-1 and Ki67 proliferation assays}

Proliferation assays were carried out ex vivo on brain metastasis tissue, in vitro on T347 cells and on the T347 cells in the BBB model system. Cell proliferation was assessed using a standard WST-1 assay. Ki-67 was assessed by IHC with mouse monoclonal anti-Ki67 (DAK0) and peroxidase-based EnVision+ kit (DAKO) (ex vivo tissue) and a TRITC-conjugated secondary antibody (Millipore). Both assays are described in detail in the supplemental methods. 


\section{Results}

$E C M$ is a key pathway in breast cancer metastasis and the ECM signalling protein ADAM22 promotes distant metastatic disease burden in vivo

RNA-seq cohorts of patient-matched primary and metastatic bone $(n=11$ patients), brain $(n=21$ patients) and liver ( $n=3$ patients) were investigated for metastasis-specific alterations. Differential gene expression functionally annotated using gene ontology demonstrated a significant enrichment for ECM and extracellular structure organisation function to all three sites (Fig. 1a, Additional file 3 Table S1). Volcano plots of differentially expressed genes were annotated by Matrisome database to further understand aberrant ECM signalling in metastasis, which confirmed the metalloproteinase family member, ADAM22, as elevated in brain metastatic tumours (Fig. 1b, Additional file 3 Table S1).
We have previously demonstrated the role for ADAM22 in ER-positive breast cancer [10]. Here we have further analysed the exome-capture RNA sequencing filtered through the Matrisome database in ER-positive patients $(n=9)$ and identified elevation in ADAM22 expression in breast cancer brain metastatic tumours (Fig. 1c, Additional file 4 Table S2), with significant gain of ADAM22 expression in ER-positive brain metastasis $(n=9$ patients, $p=0.0117$ ) (Fig. 1d). Moreover, ADAM22 expression correlated with known brain metastatic associated [28, 29] in both primary (MOCS1, $n=21, p<0.05)$ and metastatic tumours (MOCS1, COL13A1, TLR4, HBEGF and PELI2, $n=21, p<0.05)$.

Twenty of the identified ECM-related upregulated genes in ER-positive primary versus brain metastases were classified as druggable genome targets in the Drug Gene Interaction database. ADAM22 was a top ranked druggable genome target showing recurrent $(n>3$ patients)

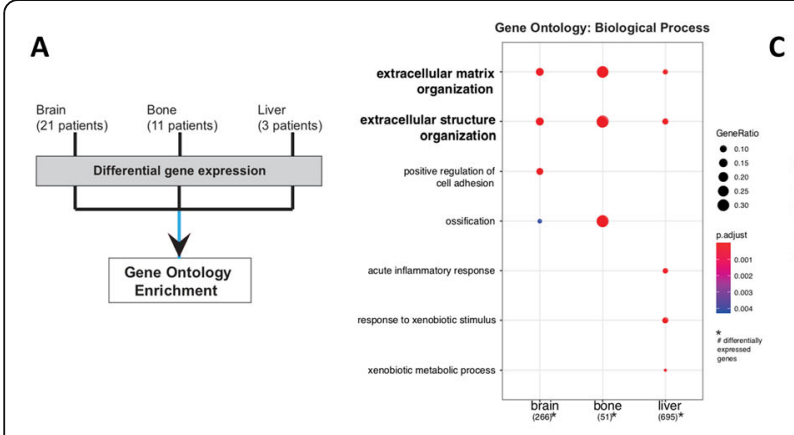

B

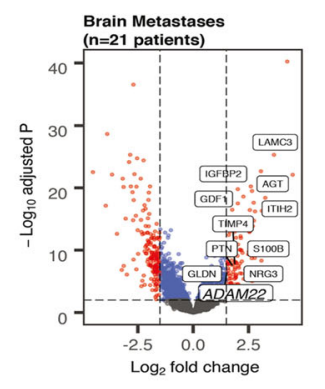

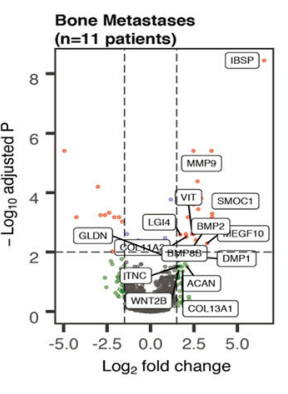

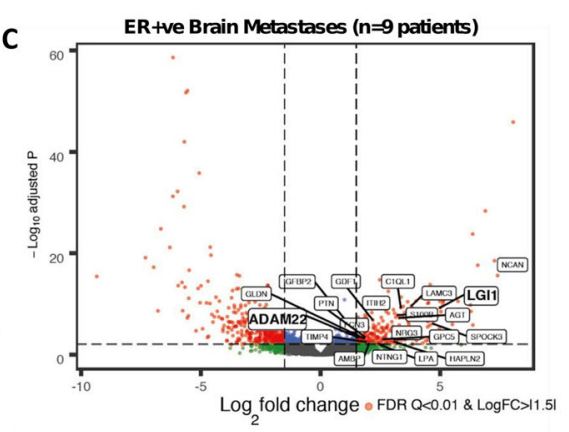

D

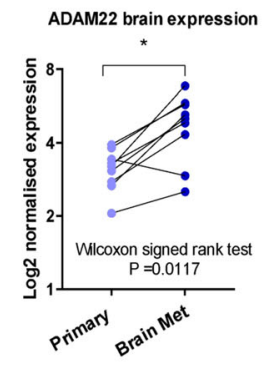

E

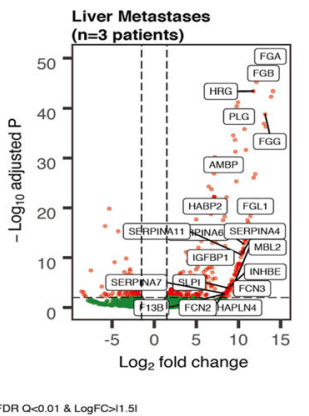

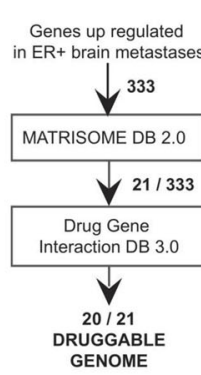

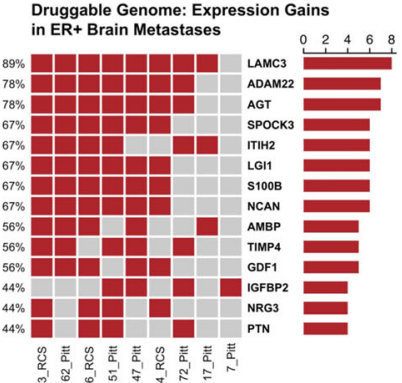

Fig. 1 ECM is a key pathway in breast cancer metastasis and the ECM signalling protein ADAM22 promotes distant metastatic disease burden in vivo. a Sequencing reads from exome capture RNAseq of patient-matched primary breast tumour with bone metastases $(n=11$ patients) and brain metastases ( $n=21$ patients) and from RNA seq of matched primary and metastatic liver ( $n=3$ patients) were mapped against human reference transcriptome GRCh38.p10. DESeq2 (exome capture RNAseq) and edgeR (RNAseq) were used to identify differential gene expression separately in primary breast tumours compared to matched brain (266), bone (51) and liver (695) metastases (log2 fold change \pm 1.5 FDR $<0.05$ ). Dotplot (right) of functional annotation of differentially expressed genes using gene ontology biological process (hypergeometric test $q$-value $<0.05$ ). b Differentially expressed genes ( $\log 2 \mathrm{FC}>1.5$, adjusted $p$ value $<0.01$ ) are displayed in the volcano plot. Genes were cross referenced against the Matrisome database to identify extracellular matrix (ECM) related genes (labelled in the volcano plot). $\mathbf{c}$ Exome-capture RNAseq of ERpositive primary breast and matched brain metastatic tissues ( $n=9$ patients, 18 samples). Differentially expressed genes (log2 FC $>1.5$, adjusted $p$ value $<0.01)$ are displayed in the volcano plot. Genes were cross referenced against the Matrisome database to identify extracellular matrix (ECM)-related genes (labelled in the volcano plot). $\mathbf{d}$ A significant increase in ADAM22 expression was found in brain metastases in patients in comparison to matched primary tissue, $n=9$, Wilcoxon matched-pairs signed-rank test ${ }^{*} p=0.0117$. e Oncoprint of recurrent ( $>3$ patients) Matrisome and Druggable Genome (Drug-Gene Interaction Database)-related gene expression gains in ER-positive brain metastases patients $(n=9)$ showed ADAM22 (78\%) as the second-ranked druggable genome in the list 
gene expression gains in ER-positive brain metastatic patients (Fig. 1e, Additional files 4 and 5 Table S2 and S3). These data raise the potential of ADAM22 as a therapeutic target in advanced ER-positive breast cancer.

\section{ADAM22 expression promotes metastatic characteristics in vivo and in vitro}

Here we examined the ability of ADAM22 to drive disease progression in an in vivo model of endocrineresistant breast cancer. Tamoxifen-resistant LY2 wildtype (WT), LY2 ADAM22 overexpressing (LY2 ADAM22 KI) and ADAM22 CRISPR/Cas9 knock-out cells (LY2 ADAM22 KO) were injected into the mammary fat pad of the NOD/SCID mice to determine the effect of ADAM22 on breast cancer disease progression in vivo (Fig. 2a). Mice implanted with ADAM22 KO cells had significantly reduced tumour volume in comparison to mice implanted with endogenous ADAM22 or ADAM22 KI cells $(p<0.0001$ and $p=0.0032$, respectively) (Fig. 2b). Furthermore, decreased tumour weight was observed between ADAM22 $\mathrm{KO}$ and WT tumours $(p<0.05)$ (Additional file 2 Fig. S3d).

Given the role of ADAM22 in breast cancer progression, the mechanism of action of the ADAM22 protein was investigated. Reverse phase protein array (RPPA) was used to explore ADAM22-dependent protein signalling in LY2 and LY2 ADAM22 KO cells in the presence of tamoxifen (4-OHT). A total of 20 proteins and phospho-proteins were found to be differentially expressed (Fig. 2c). Functional network analysis using ClueGo identified pro-proliferative and pro-metastatic pathways associated with ADAM22 expression in endocrine resistance including growth factor signalling (ERBB, VEGF and IGFR) and cell to cell communication (focal adhesion) (Fig. 2d and e). Top ranking KEGG pathways included PI3-AKT, neurotrophin signalling,

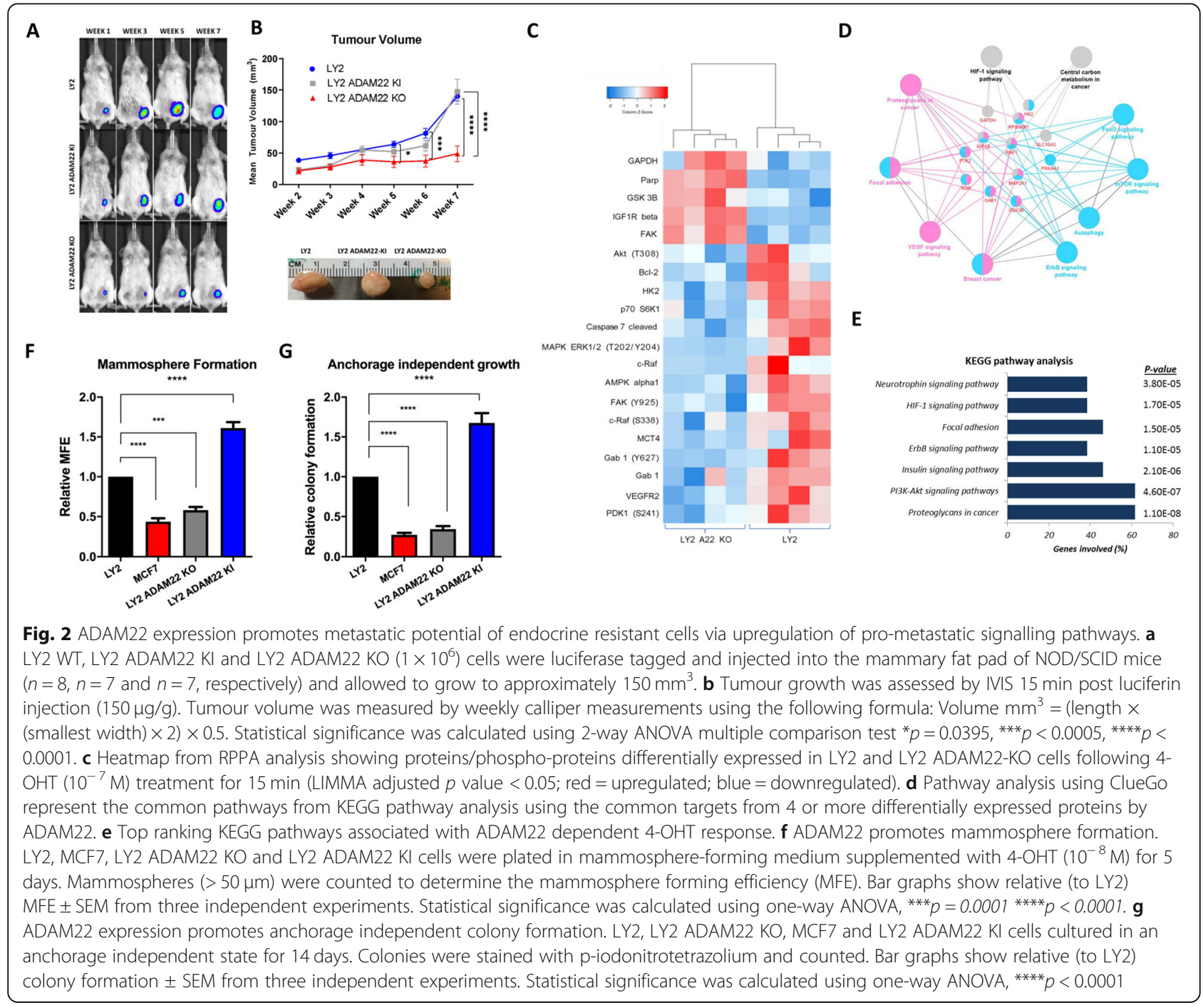


focal adhesion and ERBB signalling $(p<4.60 \mathrm{E}-07,3.80 \mathrm{E}$ $-5,1.50 \mathrm{E}-05$ and $1.10 \mathrm{E}-05$, respectively) (Fig. 2e). The functional consequences of activation of these pathways were evidenced by greater mammosphere formation and anchorage independent growth in endocrine-resistant LY2 cells in comparison to LY2 ADAM22 KO and endocrine sensitive MCF7 cells $(p<0.0001)$ (Fig. 2f and g). Moreover, elevations in mammosphere formation and anchorage independent growth were observed in LY2 ADAM22 KI cells in comparison to wild-type ADAM22 cells consistent with the characteristics of stemness and tumourigenic potential $(p=0.0002$ and $p=0.0012$, respectively) (Fig. $2 \mathrm{f}$ and g).

\section{ADAM22 is a promising drug target for metastatic breast cancer}

We have previously reported that ADAM22 protein expression in primary tumours is associated with poor disease-free survival in breast cancer patients [10]. Here we undertook ADAM22 protein target validation studies in an independent breast cancer patient cohort from a second institution ( $n=843$ patients). ADAM22 retained an association with poor disease-free survival in all breast cancer subtypes (Log-rank ( $p=0.0208$ ), Cox proportional hazard model (HR 1.59, 95\% CI is 1.04-2.43, $p=0.03$ )) (Fig. 3a) as well as in ER-positive breast cancer patients (Log-rank $(p=0.0432)$, Cox proportional hazard model (HR 1.67, 95\% CI is 1.01-2.8, $p=0.048)$ ) (Fig. 3b). Cell surface expression was detected in each of the molecular subtypes with the highest rate of expression observed in ER-positive type tumours (Additional file 2 Fig. S5a). ADAM22 expression associated with clinicopathological indicators of disease progression including tumour size $(p=0.009)$ and grade $(p=0.02)$ (Additional file 2 Fig. S5b). In line with our RNAseq studies, in matched ER-positive primary tissue and metastatic tumours (liver, brain, contralateral breast, axilla and local chest wall), where ADAM22 protein was expressed in the primary tumour, it was also expressed in the metastatic tissue (Fig. 3c, Additional file 2 Fig. S5c). To test for potential on-target side effects of a putative therapeutic targeting ADAM22, we assessed expression of the protein in healthy normal

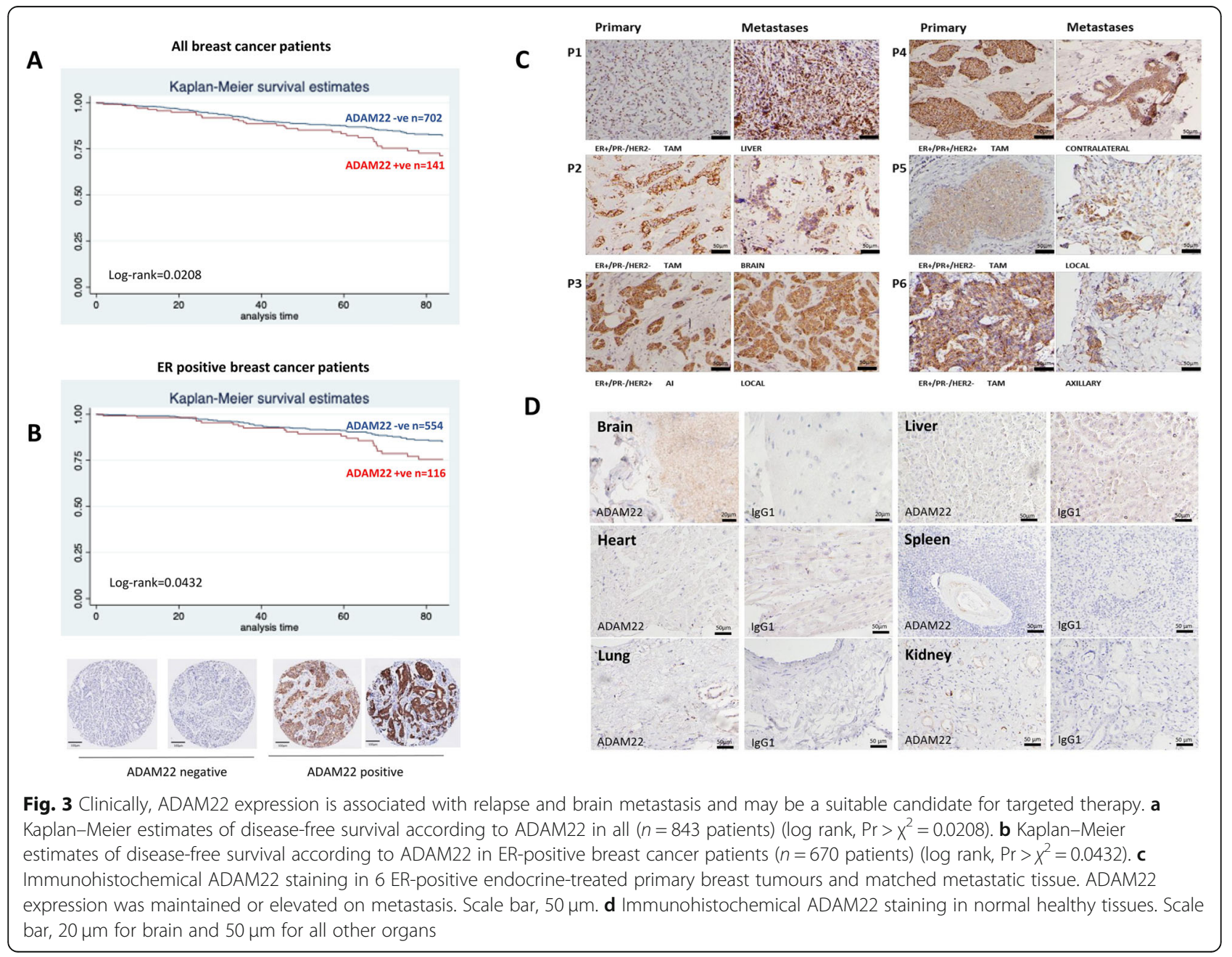


organs including the liver, brain, heart, spleen, lung and kidney. No significant expression of ADAM22 was detected in these tissues (Fig. 3d).

\section{A peptide mimetic of LGI1 binds ADAM22 and inhibits ADAM22 driven functions in vitro and ex vivo}

LGI1 is the natural ligand for ADAM22, which binds to the receptor at its disintegrin binding domain [36], a conserved region across ADAM22 isoforms 1-5. We exploited this to develop a potential therapeutic peptide to target ADAM22-positive breast cancer cells. We designed a small 21 a.a. peptide mimetic (LGI1MIM) of the disintegrin binding domain of LGI1 with a single cysteine to serine substitution (a.a. 447) to improve solubility (Fig. 4a).
The protein-peptide docking software CABS-DOCK and contact mapping modelled the LGI1MIM interaction with the disintegrin domain of ADAM22 in silico (Fig. 4b and c). LGI1MIM/ADAM22 interaction was validated in vitro using a biotin-linked LGI1MIM, which was capable of pulling ADAM22 protein from LY2 lysate (Fig. 4d). At a functional level full length LGI1 (5 nM) and LGI1MIM (10 nM) inhibited cell migration in tamoxifen-resistant LY2 cells and aromatase inhibitor-resistant LETR cells to a similar level as endocrine-sensitive MCF7 cells (Fig. 4e). LGI1MIM $(25 \mathrm{nM})$ also significantly inhibited mammopshere formation and anchorage independent growth in LY2 cells (Fig. 4f and g).

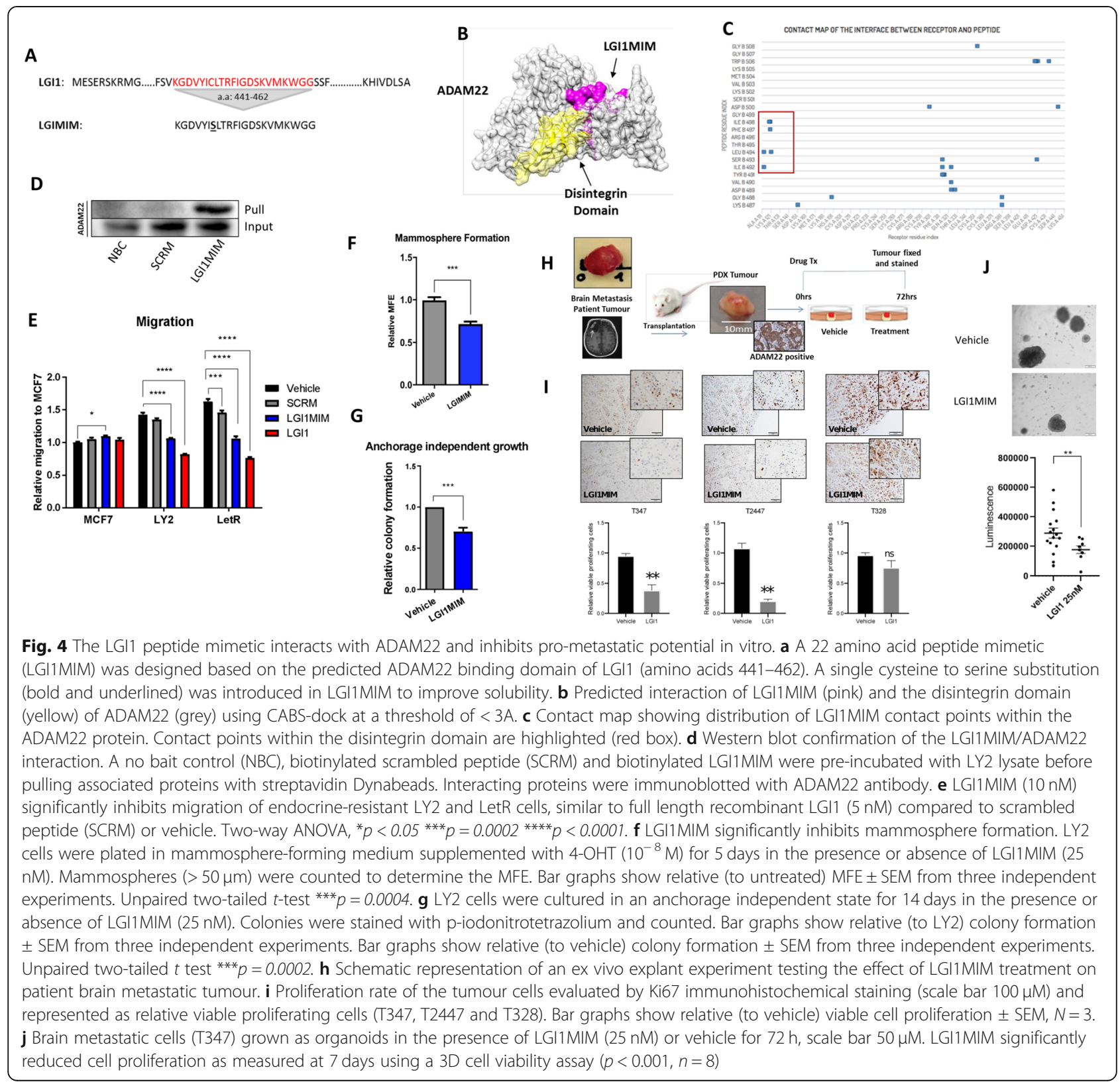


To assess the efficacy of the LGI1MIM targeting ADAM22 in patient tissue, we used endocrine-resistant ER-positive, ADAM22-positive brain metastatic tumours (T347, T2447 and T328) and a triple-negative (TNBC) brain metastatic model (T2203) (Additional file 2 Fig. S6a). The brain metastatic tissue was established and expanded in a NOD/SCID mouse and tumour-specific expression of ADAM22 was confirmed in the ex vivo tissue (Fig. 4h). Treatment with LGI1MIM for $72 \mathrm{~h}$ had substantial anti-tumour efficacy in the ex vivo endocrine-resistant and TNBC patient models (Fig. 4i and Additional file 2 Fig. S6b, respectively) as demonstrated by a decrease in proliferating tumour cells $(\mathrm{Ki} 67+)$ in comparison to vehicle-treated tumour tissue. In addition, 7-day treatment of ER-positive patient brain metastatic cells (T347 and T2447 cells) with LGI1MIM resulted in reduced organoid formation $(p<0.001, n=8$, $p<0.05, n=3$, respectively) (Fig. $4 \mathrm{j}$ and Additional file 2 Fig. S6c). Taken together, these in vitro and ex vivo studies suggest LGI1 peptide mimetic as an ADAM22specific therapeutic with anti-tumour potential.

\section{LGI1MIM is a putative therapy to treat breast cancer} brain metastasis

Drug penetration of the blood-brain barrier (BBB) represents a real clinical challenge in the development of effective treatment for brain disease. Here we have adapted a 3D biometric and biohybrid BBB model [35] to create a new patient breast cancer brain metastasis/ BBB model system to test the potential of LGI1MIM as a putative therapeutic. Lipospheres (LS) were used as a carrier system for LGI1MIM (Additional file 2 Fig. S4a). The LS were functionalised with an antibody against the transferrin receptor and loaded with the LGI1MIM peptide (LGI1MIM-LS) (Fig. 5a). Transmission electron microscope imaging (Additional file 2 Fig. S4a) revealed that LGI1MIM-LSs are characterised by unilamellar morphology and homogeneous size distribution of 100 $\mathrm{nm}$ diameter. Dynamic light scattering analysis reported a $z$-potential of $-22.7 \pm 5.1 \mathrm{mV}$, a hydrodynamic diameter of $124.6 \pm 39.4 \mathrm{~nm}$ and a polydispersity index of $0.034 \pm 0.016$ (Fig. 5b). WST-1 cell proliferation assay was carried out to evaluate the biocompatibility of the

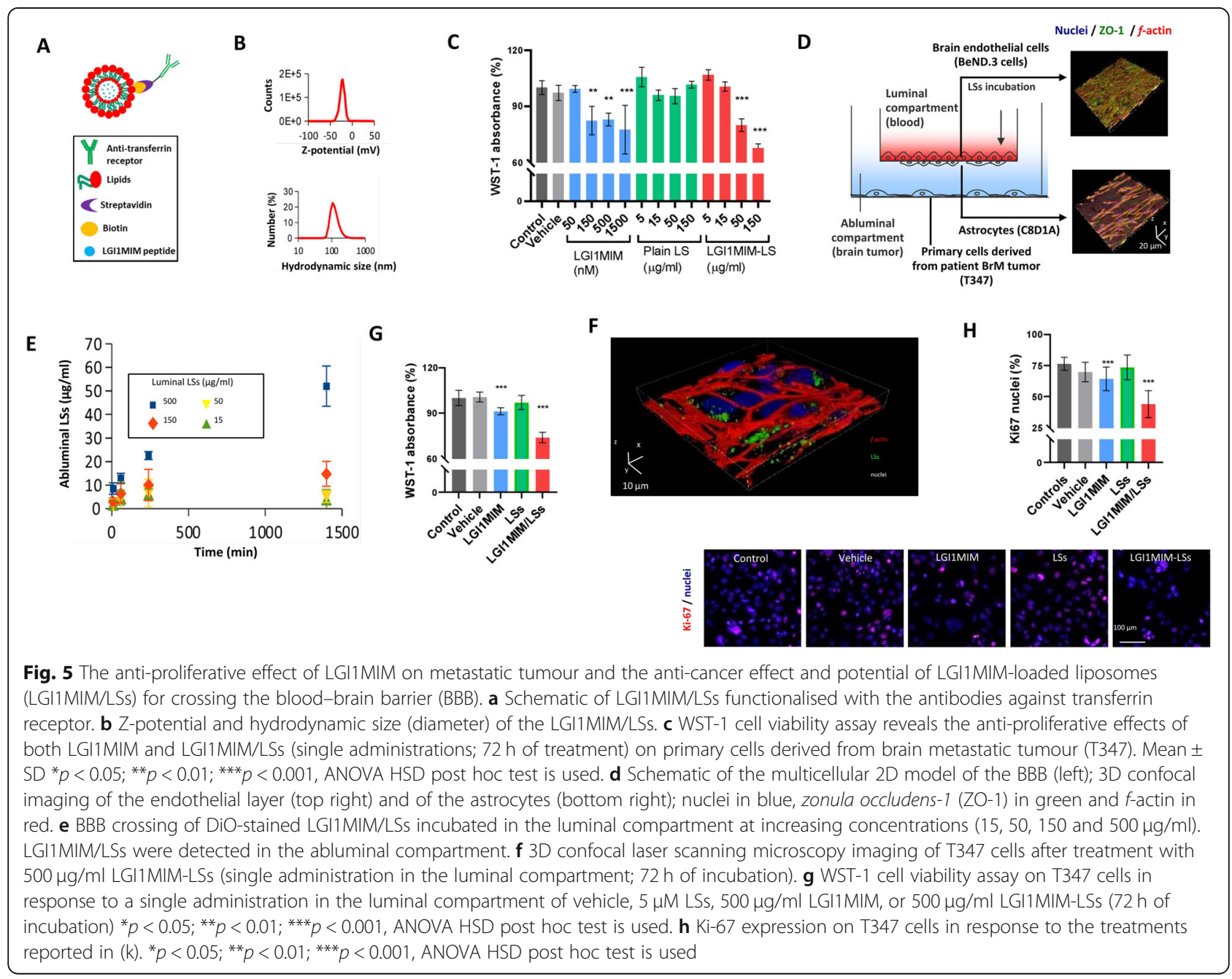


control LS-based nanovectors and the effect of LGI1MIM and LGI1MIM-LSs on patient-derived brain metastatic (T347) cell viability. LGI1MIM and LGI1MIM-LS both significantly reduced T347 cell proliferation over a range of concentrations, whereas treatment with control LS had no significant effect on proliferation (Fig. 5c).

A multicellular system was used to test the functionality of LGI1MIM-LS in the context of the BBB (Fig. 5d). Brain endothelial bEnd.3 cells were seeded to cover a porous insert, developing a biological barrier highly expressing the ZO- 1 tight junction marker at day 4 of culture (Fig. $5 \mathrm{~d}$, top right; nuclei in blue, $\mathrm{ZO}-1$ in green and f-actin in red), and C8D1A astrocytes were seeded on the abluminal side of the porous insert (Fig. $5 \mathrm{~d}$, bottom right). The bEnd. 3 cells, in combination with the C8D1A astrocytes, separated the luminal and the abluminal compartment generating a TEER of $87 \pm 9 \Omega \mathrm{cm}^{2}$. LGI1MIMLSs were found to successfully cross the BBB model (Fig. 5e). 3D confocal laser scanning microscopy imaging of T347 cells in the abluminal compartment demonstrated up-take of the DiO-stained LGI1MIM-LSs which crossed the BBB in vitro model $(t=1400 \mathrm{~min})$ (Fig. 5f).

The anti-tumour efficacy of LGI1MIM-LS in the BBB model system was assessed using the WST-1 and Ki-67 proliferation assays. The luminal compartment of the system was incubated with $500 \mu \mathrm{g} / \mathrm{ml}$ of LGI1MIM-LSs (equivalent to $50 \mu \mathrm{g} / \mathrm{ml}$ in the abluminal compartment), which did not affect the integrity of the barrier (TEER values following treatment $\left(105 \pm 9 \Omega \cdot \mathrm{cm}^{2}\right)$ versus no treatment $\left.\left(103 \pm 9 \Omega \cdot \mathrm{cm}^{2} ; p>0.05\right)\right)$. LGI1MIM and to a greater extent LGI1MIM-LS significantly reduced proliferation of breast cancer brain metastatic cells in the BBB in vitro model, with no alterations observed in the presence of control LS (Fig. 5g and h).

\section{LGI1MIM reduces metastatic burden in vivo and inhibits} formation of brain metastases

Having established the ability of LGI1MIM to cross the $\mathrm{BBB}$ and reduce tumour cell proliferation, the efficacy of LGI1MIM as a therapeutic was further evaluated in an orthotopic xenograft model of endocrine resistance. LGI1MIM tolerance was first investigated in vivo, with no observable loss of weight detected at any of the concentrations tested (Fig. 6a).

In an endocrine-resistant xenograft model, LY2 luc cells were implanted into the mammary fat pad of NOD/SCID mice and the tumours allowed to develop. At week 5, tumours were resected and animals were treated with either LGI1MIM or vehicle. LGI1MIM inhibited local disease recurrence post resection and prevented formation of distant metastatic disease of the brain and other organs including the lung, liver and bone (Additional file 2 Fig. S7a).
At a clinical level, early intervention to prevent the development of overt brain metastasis and corresponding neuronal complications is optimal. A second study was undertaken to determine the efficacy of LGI1MIM to inhibit early metastatic events (Fig. 6b). To establish baseline tumour inhibition, LY2 luc cells were implanted into the mammary fat pad of NOD/SCID mice (Fig. 6c). Tumours were allowed to develop and animals were then treated with LGI1MIM or vehicle for 6 weeks. LGI1 substantially reduced local tumour growth (mean 450 $\mathrm{mm}^{3}$ versus $150 \mathrm{~mm}^{3}$ ) (Fig. 6c), in a similar manner to that observed with the LY2-ADAM22 KO cells (Fig. 2a). To evaluate early metastatic events, we implanted a reduced number of LY2 luc cells into the mammary fat pad of the mice to maintain low primary tumour volume. Tumours were allowed to establish until palpable and animals were then treated with either LGI1MIM or vehicle (Fig. 6c). Mammary fat pad tumours were small (mean $32 \mathrm{~mm}^{3}$ ) and no difference in tumour volume was observed between the treatment groups (Fig. 6c, Additional file 2 Fig. S7b). Distant metastatic disease formation however was significantly reduced in the LGI1MIM treatment arm as determined by ex vivo IVIS imaging (Fig. $6 \mathrm{~d}$ and Additional file 2 Fig. S7c). Moreover, targeting ADAM22 with LGI1 induced consistent reductions in micro-metastatic disease burden in the brain (Fig. $6 \mathrm{e}$ and $\mathrm{f}$ ).

\section{Discussion}

Individual site-specific metastatic studies describe alterations in transcriptomic signalling, including ER expression/tyrosine kinase signalling in the brain [3], $\mathrm{CDK} / \mathrm{Rb} /$ E2F and FGFR in the bone [20] and ECM interactions in the liver $[2,35]$. Here collective analysis of differentially regulated genes in matched primary tumour and corresponding bone, brain and liver metastasis from breast cancer patients revealed $[2,37]$ that ECM and extracellular structure organisation were shared processes with the greatest enhancement on metastasis. The ADAM proteins belong to the reprolysin family and are thought to play a key role in regulating cell-cell communication [8]. We have previously described a role for ADAM22 in endocrine-resistant breast cancer, and in this study, we have further demonstrated a gain in ADAM22 expression in ER-positive brain metastases. ADAM22 can enhance cell migration and inhibit differentiation in resistant model systems and is an independent predictor of poor disease-free survival in breast cancer patients [10]. Here, ADAM22 knock-out and knock-in studies in xenograft models of endocrine resistance define a new role for ADAM22 in disease progression and metastasis.

The contribution of ADAMs to disease progression has largely been attributed to their proteolytic activity 
A

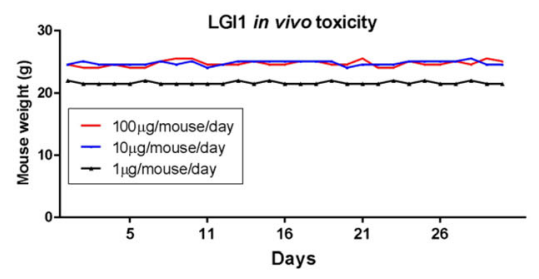

C

LGI1MIM in vivo tumour growth

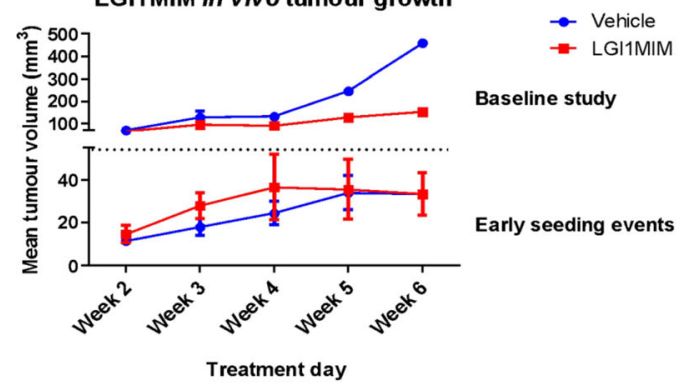

E

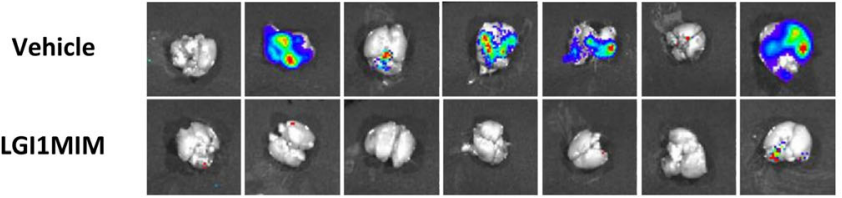

B

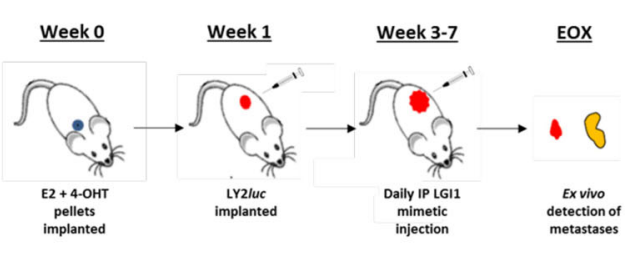

D

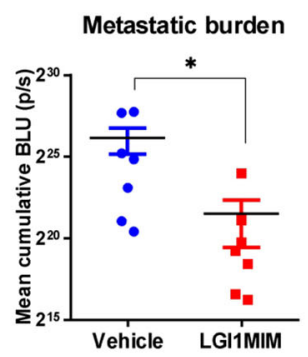

$\mathbf{F}$

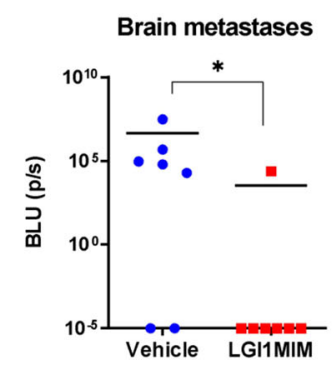

Fig. 6 The LGl1 mimetic inhibits metastatic burden in vivo. a LGI1MIM displays no toxicity in mice at low-, mid- and high-dose concentrations. NOD/SCID mice were administered a daily regimen of LGI1MIM by IP injection at three doses: $1 \mu \mathrm{g} / \mathrm{mouse} /$ day $(n=2), 10 \mu \mathrm{g} / \mathrm{mouse} / \mathrm{day}(n=2)$ and $100 \mu \mathrm{g} / \mathrm{mouse} /$ day $(n=2)$. Mouse weights were noted daily as a measure of LGI1MIM tolerability. $\mathbf{b}$ Schematic of LGI1MIM in vivo study to examine early metastatic seeding events. c Mean tumour volume in baseline study (top) determines baseline LGI1MIM tumour inhibition and the early seeding study (bottom) determines LGI1MIM effect on initial metastatic events (LGI1MIM = red; vehicle $=$ blue). Either $1 \times 10^{6}$ (baseline study) or $8 \times 10^{5}$ (early seeding study) luciferase tagged LY2 cells were implanted into the mammary fat pad of NOD/SCID mice. Mice were treated with either vehicle ( $n=2$ mice baseline study; $n=7$ mice early seeding study) or LGI1MIM at ( $100 \mu \mathrm{g} / \mathrm{mouse} /$ per day) $(n=2$ control study; $n=7$ metastatic study) for 6 weeks. LGI1MIM induced a substantial decrease in local tumour volume in the baseline study (mean $450 \mathrm{~mm}^{3}$ versus $150 \mathrm{~mm}^{3}$, calliper measurement), whereas no alteration was detected in the early metastatic seeding model. $\mathbf{d}$ Cumulative metastatic burden in vehicle- and LGI1MIM-treated mice. LGI1MIM significantly reduces metastatic burden (as measured by BLU $\log ^{2}$ p/s). Two tailed Mann-Whitney test* $p=0.0111$. e The ex vivo luciferase activity from brain metastases in vehicle-treated (top) and LGI1MIM-treated (bottom) mice. $\mathbf{f}$ Brain metastatic burden, BLU $\log ^{2} \mathrm{p} / \mathrm{s}$, was significantly reduced in LGI1MIM treated mice (red; $n=7$ mice) versus vehicle control (blue; $n=7$ mice). Two-tailed Mann-Whitney test ${ }^{*} p=0.0373$

mediated through a functional metalloproteinase domain [14, 38]. ADAM22 however lacks an active metalloproteinase site and has no proteolytic activity [14]. Though there has been little study to date on the non-catalytic function of ADAM proteins in cancer, a role for ADAM22 in cell adhesion and spreading in conjunction with the 14-3-3 family of signalling proteins has been described [39-41]. Here an RPPA approach was used to assess ADAM22dependent signalling in endocrine-resistant breast cancer. Cell-to-cell communication and growth factor pathway activation, including HER2 signalling, VEGF and IGFR, were observed as key ADAM22-associated networks. The significance of an ADAM17-EGFR axis in mammary gland development and cancer has previously been described [42]. ADAM22 may use similar signalling mechanisms to drive metastatic processes including stem-like activity and anchorage independent growth observed in this study, potentially through its cysteine-rich/EGF-like domains.

We assessed the potential of ADAM22 as a therapeutic target. Robust cell surface ADAM22 protein was found in approximately $17 \%$ of primary breast cancer tissues across all of the molecular sub-types. Elevation of ADAM22 transcript was observed in metastatic tumours in comparison to matched primary tissue, particularly in breast to brain metastasis, as evidenced in both transcript and protein. For any potential drug target, it is necessary to assess the on-target side effects [43]. Here potential ADAM22 ontarget side effect was considered low, as insignificant 
ADAM22 protein expression was observed in normal healthy organs in comparison to primary and metastatic breast cancer tissue. However more in-depth toxicity studies will need to be undertaken to establish the full safety profile of ADAM22 targeted therapies.

ADAM22 can interact with extracellular proteins to alter cell processes. LGI1 has been shown to act as a specific extra-cellular ligand for ADAM22 to regulate synaptic transmission through stabilisation of the AMPA/stargazin complex [36]. The LGI1-ADAM22 ligand/receptor complex has been suggested as a potential therapeutic target to treat synaptic disorders in the nervous system [13]. In cancer, a tumour suppressor role for LGI1 has been described in glioblastoma and neuroblastoma, and it has been shown that LGI1 may play a role in impairing proliferation and survival in HeLa cells [44-46]. We have previously reported LGI1-induced inhibition of cell migration in endocrine-resistant breast cancer cells [10]. Furthermore, in this study, analysis of metastatic dominant ECM genes in a cohort of matched primary and metastatic tumours revealed ADAM22 as a top member of the druggable genome. These data raise the possibility of LGI1/ADAM22 as a therapeutic complex to treat advanced breast cancer.

There has been an increased interest in use of therapeutic peptides as they have demonstrated to be highly selective and efficacious, with low cytotoxicity [47]. Of interest, therapeutic peptides have shown better efficacy in comparison to antibody-based therapies in penetrating blood-brain barrier and for treatment of brain cancers $[48,49]$. To exploit LGI1 as a therapeutic, we designed a small peptide mimetic against the ligand binding pocket of LGI1 in the disintegrin binding domain of ADAM22. Successful binding was confirmed in silico and in vitro. At a functional level, the peptide had similar effect as full length LGI1, halting cell migration of aggressive endocrine-resistant cells to a comparable level of that observed in endocrine-sensitive MCF7 cells. To further asses the efficacy of LGI in targeting ADAM22 in breast cancer patient samples, an ADAM22-positive brain metastatic patient model was used [3]. Treatment of this patient ex vivo model with LGI1MIM demonstrated an antitumourigenic efficacy as evidenced by reductions in the proliferation marker Ki67.

Treatment of breast cancer brain metastasis is a significant challenge, in particular with regard to ensuring successful drug penetration of the BBB [50]. Given the elevated levels of ADAM22 in breast cancer brain metastatic tumours and the efficacy of LGI1MIM in inhibiting early metastatic events, we evaluated the potential of LGI1/ADAM22 complex as a new therapeutic strategy in breast cancer-BBB model systems. Model systems to test new treatments are challenging, and none is without limitation; here we test LGI1 in a patient-derived system.
We adapted a 3D biometric and biohybrid system [35] to create a multi-cellular breast cancer BBB model, encompassing endothelial cells, astrocytes and patientderived brain metastatic tumour cells. This model recapitulates the dynamics of the BBB in vivo and has the potential for assessing the efficacy of new brain cancer therapeutics beyond this study. In this model system LGI1MIM and in particular LGI1MIM-LS were found to successfully cross the $\mathrm{BBB}$ and inhibit tumour cell proliferation.

The ability of LGI1MIM to target ADAM22 either in the primary or metastatic site to inhibit the development of disease progression was demonstrated in vivo. Given that early intervention is crucial in combating progression to metastasis [51], further clinical utility of the drug was assessed in the micro-metastatic setting. The LGI1MIM prevented early progression of breast cancer brain metastasis in our endocrine-resistant breast cancer xenograft model. Though these in vivo studies provide considerable evidence of LGI1MIM as a putative therapeutic to treat the development of brain metastasis, further studies investigating drug metabolism as well as ontarget side effects in normal brain tissue are required.

\section{Conclusion}

Recurrent elevations in ECM organisation networks are observed on breast cancer metastasis. Data presented in this study suggest that targeting the ECM signalling protein ADAM22 may represent a new therapeutic strategy to treat breast cancer brain metastasis.

\section{Supplementary information}

Supplementary information accompanies this paper at https://doi.org/10. 1186/s12916-020-01806-4.

Additional file 1: Supplemental Methods.

Additional file 2: Figure S1. Confirmation of LY2 ADAM22 CRISPR knockout cell line. (a) RFP expression confirming CRISPR /Cas9 mediated double stranded break and insertion of an RFP tagged HDR plasmid (b) FACS gating strategy based on high RFP expression to single cell sort CRISPR/Cas9 ADAM22 KO cells (right) from LY2 WT cells (left) (c) Confirmation of ADAM22 gene silencing in an LY2 ADAM22 KO clone (Clone $\mathrm{H}(\mathrm{C}-\mathrm{H}))$ by PCR and Western blot analysis $(n=4) .{ }^{* * *} p<0.0005$ (d) Genotyping of Clone $\mathrm{H}$ using primers which flank the ADAM22 CRIS PR sgRNA sites 1 and 3 (left) and sgRNA 2 (right). Clone H contains a homozygous deletion at cut site $1 \& 3$ and a heterozygous HDR insertion at cut site 2. (e) Sanger sequencing of the homozygous $58 \mathrm{bp}$ deletion in Clone $\mathrm{H}$ (red) flanked by the sgRNA 1 and 3 cut sites (lilac) in LY2 WT cells. Figure S2. Confirmation of LY2 lentiviral ADAM22 knock in (KI) cell line. (a) GFP expression confirming successful transduction of LY2 cells with lentiviral ADAM22 particles. (b) FACS gating strategy based on high GFP expression to purify LY2 ADAM22 KI cells (right) from LY2 WT cells (left). (c) Confirmation of ADAM22 gene overexpression in the LY2 lentiADAM22 KI cell line. ${ }^{*} p<0.05$. (d) Western blot confirmation of ADAM22 overexpression in LY2 lentiADAM22 cells versus LY2 WT cells. Figure S3. ADAM $22 \mathrm{KO}, \mathrm{Kl}$ and $\mathrm{WT}$ cells. (a) ADAM22 protein expression in each biological replicate used for the RPPA study. (b) IVIS imaging of luciferase activity in LY2 luc, Clone H luc (ADAM22 KO) and lentiA22 luc (ADAM22 Kl) cells in vitro after treatment with $15 \mu \mathrm{g} / \mathrm{ml}$ of luciferin. (c) 
Luciferase activity was comparable across each cell line with respect to cell number. (d) Tumour weight was significantly reduced in LY2 ADAM22 KO tumours versus LY2 WT. Tumour weight \pm SEM, Unpaired Mann Whitney two tailed t test ${ }^{*} p<0.05$. Figure S4. Quantification of LGIMIM peptide in LSs. (a) Transmission electron microscopy (TEM) imaging of LGI1MIM-LSs and SDS-page stained with Coomassie Blue of LGI1MIM (6.0, 2.0, 0.7 and $0.2 \mu \mathrm{g})$, empty LSs $(5 \mathrm{mg} / \mathrm{ml}$ ) (b) Calibration curve reporting the band intensities (pixel values) for different amounts of LGI1MIM. Figure S5. ADAM22 clinical data. (a) ADAM22 expression was examined in a cohort of 843 breast cancer patients and the percentage of ADAM22-positive is stated in each of the breast cancer subtypes as well as in patients with recurrent disease. (b) Associations of ADAM22 in TMA of breast cancer patients with clinicopathologic variables using chi-square test. (C) Clinical molecular status, treatment data and $\mathrm{H}$-score from primary and metastatic patient tumours. Figure S6. A peptide mimetic of LG11 inhibits ADAM22 driven functions in vitro and ex vivo (a) Clinical molecular status of patient tumours. (b) Proliferation rate of the tumour cells from a triple negative tumour (T2203) in an ex vivo explant

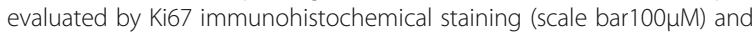
represented as relative viable proliferating cells. (b) Brain metastatic cells from an ER positive patient tumour (T2447) grown as organoids in the presence of LGI1MIM ( $25 \mathrm{nM}$ ) or vehicle for $72 \mathrm{~h}$, scale bar $50 \mu \mathrm{M}$. LGI1MIM significantly reduced cell proliferation as measured at 7 days using a $3 \mathrm{D}$ cell viability assay $(P=0.0373, n=3)$. Figure S7. LGI1MIM inhibits disease progression in endocrine resistant xenograft models. (a) Local and distant metastatic disease were assessed in a resection endocrine resistant xenograft model. $1 \times 10^{6}$ luciferase tagged LY2 cells were injected into the left inguinal mammary fat pad of NOD/SCID mice Tumours were allowed to form and at week 5 , primary tumours were surgically removed. Mice were treated either with vehicle ( $n=3$ mice) (top) or LGI1MIM (100 $\mu \mathrm{g} /$ mouse/day) ( $n=2$ mice) (bottom) for 6 weeks. Tumour growth was monitored using calliper measurement. Mice were culled at the end of the experimental period (15 weeks) or once the tumour reached $500 \mathrm{~mm} 3$. Local (in vivo) and distant organ specific (ex vivo) recurrences were examined using an IVIS imaging system. (b) Weekly IVIS readings of local tumour burden in early seeding study. $800 \times 10^{5}$ luciferase tagged LY2 cells were implanted into the mammary fat pad of NOD SCID mice. Mice were treated with either vehicle ( $n=7$ mice metastatic study) or LGI1MIM at ( $100 \mu \mathrm{g} / \mathrm{mouse} /$ per day) ( $n=7$ metastatic study). No differences in tumour cells (mean BLU (p/s) scores) was detected in vehicle versus LGI1MIM treatment groups. (c) Representative image of ex vivo luciferase activity from Lung, bone, liver and brain metastases in vehicle treated (left) and LGI1MIM treated (right) mice. No significant differences in metastatic burden were observed in liver and lung, Bone and brain showed reduced metastatic burden in LGIIMIM treated mice compared to vehicle treated mice, BLU $\log 2 \mathrm{p} / \mathrm{s},(n=7$ mice) Two tailed Mann Whitney test** $p=0.007$, bone and ${ }^{*} p=0.0373$ brain

Additional file 3: Table S1. Differential gene expression (log2FC +/1.5 adjusted pval $<0.05$ ) metastases vs primary sets used as input for Gene Ontology Enrichment Testing with matrisomeDB annotation.

Additional file 4: Table S2. Differentially expressed protein coding genes $(\log 2 \mathrm{FC}+/-1.5)$ in ER+ primary breast and matched brain metastatic tumours ( $N=9$ patients).

Additional file 5: Table S3. ER+ subtype patient specific fold-changes and expression gains in drug targetable matrisome associated genes ( $N=9$ patients).

\section{Acknowledgements}

We are thankful to the patients who generously provided tumour tissue for our studies and to the surgical, pathology and tissue bank colleagues for their substantial assistance and support. We would like to thank Dr. Simone Lauciello (Istituto Italiano di Tecnologia), for the transmission electron microscopy imaging of the liposomes.

\section{Authors' contributions}

Study concept and design (S.Ch., B.D, G.C.L.S.Y.); acquisition, analysis, or interpretation of data (B.D., S.Ch., S.C., D.V., A.M, R.M., S.P., F.T.B., J.B., C.B., C.M., M. B., N.P., S.O.,A.V.L.,L.S.Y); bioinformatic analyses (N.C., S.M., N.P.); provision of administrative, technical or material support (N.P., P.O'H., F.B., K.S., F.T.B, S.C.,
K.B., A.M.H., S.O., A. L., A.D.K.H.); drafting of the manuscript (S.Ch, D.V, F.T.B., A.M., G.C., L.S.Y.); critical revision of the manuscript (all authors); study supervision (S.Ch., L.S.Y.). All authors have read and approved the manuscript.

\section{Funding}

We kindly acknowledge the funding support from SFI Pfizer Biotherapeutics Award (LY, SC), Irish Cancer Society Collaborative Cancer Research Centre grant, CCRC13GAL (LY), Science Foundation Ireland Investigator Award 12/IA 1294 (LY), Breast Cancer Ireland Programme Grant (LY), EU- FP7-IAPP (LY), Breast Cancer NOW grant, 2018JulPR1094 (LY, DV), SFI Strategic Partnership Programme POI, 18/SPP/5322 (LY), Breast Cancer NOW Fellowship 2019AugSF1310 (DV), and Science Foundation Ireland Frontiers Award (AH).

\section{Availability of data and materials}

All data will be made available and uploaded to the relevant publicly accessible databases.

\section{Ethics approval and consent to participate}

All patient clinical material was collected under the clinical trial NCT01840293 (https://clinicaltrials.gov, CTRIAL-IE 09/07) following ethical approval from Beaumont Hospital Medical Research Ethics Committee. All patients were fully consented onto the trial. All animal experiments were performed in accordance with the EU Directive 210/63/EU and were reviewed and approved by Research Ethics Committee, RCSI under licence from The Health Products Regulatory Authority. Experiments were undertaken in accordance with the Arrive Guidelines where appropriate.

\section{Consent for publication}

Not applicable.

\section{Competing interests}

The authors declare that they have no competing interests. LY has previously had collaborative funding with Pfizer from Science Foundation Ireland.

\section{Author details}

${ }^{1}$ Endocrine Oncology Research Group, Department of Surgery, Royal College of Surgeons in Ireland, Dublin 2, Ireland. ${ }^{2}$ Smart Bio-Interfaces, Istituto Italiano di Tecnologia, Scuola Superiore Sant'Anna, Pontedera, Italy. ${ }^{3}$ Women's Cancer Research Centre, Magee-Women's Research Institute, University of Pittsburgh Cancer Institute, University of Pittsburgh, Pittsburgh, PA, USA. ${ }^{4}$ Department of Neurosurgery, National Neurosurgical Centre, Beaumont Hospital, Dublin, Ireland. ${ }^{5}$ Department of Neuropathology, Beaumont Hospital, Dublin, Ireland. ${ }^{6}$ Department of Pathology, Royal College of Surgeons in Ireland, Dublin, Ireland. 'Department of Surgery, Royal College of Surgeons in Ireland, Dublin, Ireland. ${ }^{8}$ Data Science Centre, Royal College of Surgeons in Ireland, Dublin, Ireland. ${ }^{9}$ The Biorobotics Institute, Scuola Superiore Sant'Anna, Pontedera, Italy. ${ }^{10}$ Department of Surgery, Beaumont Hospital, Dublin, Ireland.

Received: 16 June 2020 Accepted: 2 October 2020

Published online: 19 November 2020

\section{References}

1. Mehlen P, Puisieux A. Metastasis: a question of life or death. Nat Rev Cancer. 2006;6(6):449-58.

2. McBryan J, Fagan A, McCartan D, Bane FT, Vareslija D, Cocchiglia S, et al. Transcriptomic profiling of sequential tumors from breast cancer patients provides a global view of metastatic expression changes following endocrine therapy. Clin Cancer Res. 2015;21(23):5371-9.

3. Vareslija D, Priedigkeit N, Fagan A, Purcell S, Cosgrove N, O'Halloran PJ, et al. Transcriptome characterization of matched primary breast and brain metastatic tumors to detect novel actionable targets. J Natl Cancer Inst. 2019;111(4):388-98.

4. Mead TJ, Apte SS. ADAMTS proteins in human disorders. Matrix Biol. 2018; 71-72:225-39.

5. Turner SL, Blair-Zajdel ME, Bunning RA. ADAMs and ADAMTSs in cancer. Br J Biomed Sci. 2009:66(2):117-28.

6. Edwards DR, Handsley MM, Pennington CJ. The ADAM metalloproteinases Mol Asp Med. 2008;29(5):258-89.

7. Reiss K, Saftig P. The "a disintegrin and metalloprotease" (ADAM) family of sheddases: physiological and cellular functions. Semin Cell Dev Biol. 2009; 20(2):126-37. 
8. Murphy G. The ADAMs: signalling scissors in the tumour microenvironment. Nat Rev Cancer. 2008;8(12):929-41.

9. Duffy MJ, Mullooly M, O'Donovan N, Sukor S, Crown J, Pierce A, et al. The ADAMs family of proteases: new biomarkers and therapeutic targets for cancer? Clin Proteomics. 2011:8(1):9.

10. McCartan D, Bolger JC, Fagan A, Byrne C, Hao Y, Qin L, et al. Global characterization of the SRC-1 transcriptome identifies ADAM22 as an ERindependent mediator of endocrine-resistant breast cancer. Cancer Res. 2012;72(1):220-9.

11. Li J, Lu M, Jin J, Lu X, Xu T, Jin S. miR-449a suppresses tamoxifen resistance in human breast cancer cells by targeting ADAM22. Cell Physiol Biochem. 2018;50(1):136-49.

12. Liu $H$, Shim $A H, H e X$. Structural characterization of the ectodomain of a disintegrin and metalloproteinase-22 (ADAM22), a neural adhesion receptor instead of metalloproteinase: insights on ADAM function. J Biol Chem. 2009; 284(42):29077-86.

13. Fukata $Y$, Lovero KL, Iwanaga $T$, Watanabe A, Yokoi N, Tabuchi K, et al. Disruption of LGl1-linked synaptic complex causes abnormal synaptic transmission and epilepsy. Proc Natl Acad Sci U S A. 2010;107(8):3799-804.

14. Bolger JC, Young LS. ADAM22 as a prognostic and therapeutic drug target in the treatment of endocrine-resistant breast cancer. Vitam Horm. 2013;93: 307-21

15. Chernova OB, Somerville RP, Cowell JK. A novel gene, LGl1, from 10q24 is rearranged and downregulated in malignant brain tumors. Oncogene. 1998; 17(22):2873-81.

16. Kodack DP, Askoxylakis V, Ferraro GB, Fukumura D, Jain RK. Emerging strategies for treating brain metastases from breast cancer. Cancer Cell. 2015;27(2):163-75

17. Bronzert DA, Greene GL, Lippman ME. Selection and characterization of a breast cancer cell line resistant to the antiestrogen LY 117018. Endocrinology. 1985;117(4):1409-17

18. Ward E, Vareslija D, Charmsaz S, Fagan A, Browne AL, Cosgrove N, et al. Epigenome-wide SRC-1-mediated gene silencing represses cellular differentiation in advanced breast cancer. Clin Cancer Res. 2018;24(15):3692703.

19. Vareslija D, McBryan J, Fagan A, Redmond AM, Hao Y, Sims AH, et al. Adaptation to Al therapy in breast cancer can induce dynamic alterations in ER activity resulting in estrogen-independent metastatic tumors. Clin Cancer Res. 2016;22(11):2765-77

20. Priedigkeit N, Watters RJ, Lucas PC, Basudan A, Bhargava R, Horne W, et al. Exome-capture RNA sequencing of decade-old breast cancers and matched decalcified bone metastases. JCl insight. 2017;2(17).

21. Patro R, Duggal G, Love MI, Irizarry RA, Kingsford C. Salmon provides fast and bias-aware quantification of transcript expression. Nat Methods. 2017; 14(4):417-9.

22. Love Ml, Huber W, Anders S. Moderated estimation of fold change and dispersion for RNA-seq data with DESeq2. Genome Biol. 2014;15(12):550.

23. Naba A, Clauser KR, Ding H, Whittaker CA, Carr SA, Hynes RO. The extracellular matrix: tools and insights for the "omics" era. Matrix Biol. 2016; 49:10-24.

24. Robinson MD, McCarthy DJ, Smyth GK. edgeR: a Bioconductor package for differential expression analysis of digital gene expression data. Bioinformatics. 2010;26(1):139-40.

25. Yu G, Wang LG, Han Y, He QY. clusterProfiler: an R package for comparing biological themes among gene clusters. OMICS. 2012;16(5):284-7.

26. Cotto KC, Wagner AH, Feng YY, Kiwala S, Coffman AC, Spies G, et al. DGldb 3.0: a redesign and expansion of the drug-gene interaction database. Nucleic Acids Res. 2018;46(D1):D1068-D73.

27. Gu Z, Eils R, Schlesner M. Complex heatmaps reveal patterns and correlations in multidimensional genomic data. Bioinformatics. 2016;32(18): 2847-9.

28. Bos PD, Zhang XH, Nadal C, Shu W, Gomis RR, Nguyen DX, et al. Genes that mediate breast cancer metastasis to the brain. Nature. 2009:459(7249):1005-9.

29. Kanojia D, Morshed RA, Zhang L, Miska JM, Qiao J, Kim JW, et al. BIII-Tubulin regulates breast cancer metastases to the brain. Mol Cancer Ther. 2015; 14(5):1152-61.

30. Shaw FL, Harrison H, Spence K, Ablett MP, Simoes BM, Farnie G, et al. A detailed mammosphere assay protocol for the quantification of breast stem cell activity. J Mammary Gland Biol Neoplasia. 2012;17(2):111-7.
31. Browne AL, Charmsaz S, Vareslija D, Fagan A, Cosgrove N, Cocchiglia S, et al. Network analysis of SRC-1 reveals a novel transcription factor hub which regulates endocrine resistant breast cancer. Oncogene. 2018;37(15):2008-21.

32. McBryan J, Theissen SM, Byrne $C_{1}$ Hughes $E_{1}$ Cocchiglia S, Sande $S_{\text {, et al. }}$ Metastatic progression with resistance to aromatase inhibitors is driven by the steroid receptor coactivator SRC-1. Cancer Res. 2012;72(2):548-59.

33. Charmsaz S, Hughes E, Bane FT, Tibbitts P, Mcllroy M, Byrne C, et al. S100beta as a serum marker in endocrine resistant breast cancer. BMC Med. 2017;15(1):79.

34. Sachs N, de Ligt J, Kopper O, Gogola E, Bounova G, Weeber F, et al. A living biobank of breast cancer organoids captures disease heterogeneity. Cell. 2018;172(1-2):373-86.e10.

35. Marino A, Almici E, Migliorin S, Tapeinos C, Battaglini M, Cappello V, et al. Piezoelectric barium titanate nanostimulators for the treatment of glioblastoma multiforme. J Colloid Interface Sci. 2019;538:449-61.

36. Fukata Y, Adesnik H, Iwanaga T, Bredt DS, Nicoll RA, Fukata M. Epilepsyrelated ligand/receptor complex LGI1 and ADAM22 regulate synaptic transmission. Science. 2006;313(5794):1792-5.

37. Kimbung S, Johansson I, Danielsson A, Veerla S, Egyhazi Brage S, Frostvik Stolt M, et al. Transcriptional profiling of breast cancer metastases identifies liver metastasis-selective genes associated with adverse outcome in luminal a primary breast cancer. Clin Cancer Res. 2016;22(1):146-57.

38. Conrad C, Benzel J, Dorzweiler K, Cook L, Schlomann U, Zarbock A, et al. ADAM8 in invasive cancers: links to tumor progression, metastasis, and chemoresistance. Clin Sci (Lond). 2019;133(1):83-99.

39. Jones DH, Ley S, Aitken A. Isoforms of 14-3-3 protein can form homo- and heterodimers in vivo and in vitro: implications for function as adapter proteins. FEBS Lett. 1995;368(1):55-8.

40. Zhu P, Sang Y, Xu H, Zhao J, Xu R, Sun Y, et al. ADAM22 plays an important role in cell adhesion and spreading with the assistance of 14-3-3. Biochem Biophys Res Commun. 2005;331(4):938-46.

41. Zhu P, Sang Y, Xu R, Zhao J, Li C, Zhao S. The interaction between ADAM22 and 14-3-3beta. Sci China C Life Sci. 2002;45(6):577-82.

42. Sternlicht MD, Sunnarborg SW. The ADAM17-amphiregulin-EGFR axis in mammary development and cancer. J Mammary Gland Biol Neoplasia. 2008; 13(2):181-94.

43. Gabellini N, Masola V, Quartesan S, Oselladore B, Nobile C, Michelucci R, et al. Increased expression of LGl1 gene triggers growth inhibition and apoptosis of neuroblastoma cells. J Cell Physiol. 2006;207(3):711-21.

44. Bornstein GG. Antibody drug conjugates: preclinical considerations. AAPS J. 2015;17(3):525-34.

45. Gabellini N, Masola V. Expression of LGl1 impairs proliferation and survival of HeLa cells. Int J Cell Biol. 2009;2009:417197.

46. Kunapuli P, Kasyapa CS, Hawthorn L, Cowell JK. LGI1, a putative tumor metastasis suppressor gene, controls in vitro invasiveness and expression of matrix metalloproteinases in glioma cells through the ERK1/2 pathway. J Biol Chem. 2004;279(22):23151-7.

47. Fosgerau K, Hoffmann T. Peptide therapeutics: current status and future directions. Drug Discov Today. 2015;20(1):122-8.

48. Hyvonen M, Enback J, Huhtala T, Lammi J, Sihto H, Weisell J, et al. Novel target for peptide-based imaging and treatment of brain tumors. Mol Cancer Ther. 2014;13(4):996-1007.

49. Marqus S, Pirogova E, Piva TJ. Evaluation of the use of therapeutic peptides for cancer treatment. J Biomed Sci. 2017;24(1):21.

50. Liu MC, Cortes J, O'Shaughnessy J. Challenges in the treatment of hormone receptor-positive, HER2-negative metastatic breast cancer with brain metastases. Cancer Metastasis Rev. 2016;35(2):323-32.

51. Hu Y, Yu X, Xu G, Liu S. Metastasis: an early event in cancer progression. J Cancer Res Clin Oncol. 2017:143(5):745-57.

\section{Publisher's Note}

Springer Nature remains neutral with regard to jurisdictional claims in published maps and institutional affiliations. 